\title{
A Comparison of the Psychological Outcomes of Cosmetic Surgical Procedures
}

\author{
Nicki A. Dowling ${ }^{1}$, Alun C. Jackson ${ }^{1,2}$ and Roberta J. Honigman ${ }^{3}$ \\ ${ }^{1}$ The University of Melbourne, Melbourne, Australia \\ ${ }^{2}$ Centre on Behavioural Health, University of Hong Kong \\ ${ }^{3}$ St. Vincent's Hospital, Melbourne, Australia
}

\begin{abstract}
Studies of psychological outcomes of cosmetic surgery generally provide low standards of evidence due to significant methodological limitations and few studies have explored the outcomes of surgery types other than breast reduction or have compared post-operative outcomes for different surgery types. This study therefore aimed to explore the psychological outcomes and post-operative satisfaction of cosmetic surgery and compare the outcomes and satisfaction of participants undergoing different procedures. Surveys were administered to a sample of 142 elective cosmetic surgery patients prior to surgery and six months after surgery. The surveys included standardised measures of body image (appearance evaluation, appearance orientation, body areas satisfaction), self-esteem, and mental health (psychiatric disturbance, anxiety, depression, dysmorphic concerns). The findings revealed high rates of post-operative satisfaction and significant improvements in several dimensions of body image (appearance evaluation and body area satisfaction) and mental health (anxiety, depression, and dysmorphic concerns), but not self-esteem. Breast augmentation patients reported significantly higher improvement on appearance evaluation, significantly higher ratings of how likely they thought it was that they had achieved, or would achieve, what they were looking for in having the procedure, and a trend to report that the overall result of surgery was better than or as expected than rhinoplasty patients. It is recommended that future research clarifies the degree to which self-esteem and indices of mental health improve after cosmetic surgery and fully establishes the impact of different procedures on psychological outcomes. Level of Evidence. Therapeutic Study, Level II.
\end{abstract}

Keywords: Cosmetic surgery; plastic surgery; breast augmentation; rhinoplasty.

\section{Introduction}

Studies of outcomes for cosmetic surgery generally reveal high rates of patient satisfaction (Honigman, Jackson, \& Dowling, 2011; Klassen, Jenkinson, Fitzpatrick, \& Goodacre, 1996; von Soest, Kvalem, Roald, \& Skolleborg, 2009). However, patient satisfaction does not necessarily indicate improved psychological functioning (Cook, Rosser, \& Salmon, 2006; Honigman, Phillips, \& Castle,
2004). Two systematic reviews have derived somewhat different conclusions regarding improvements in the psychological outcomes of cosmetic surgery.

Honigman et al. (2004) conducted a review of 37 retrospective and prospective studies published from 1960 to 2002. Inclusion criteria included pre-operative and postoperative measures of psychological outcomes (such as distress, body image,

Copyright (C) 2013 Nicki A. Dowling, Alun C. Jackson and Roberta J. Honigman. This is an open access article distributed under the Creative Commons Attribution License unported 3.0, which permits unrestricted use, distribution, and reproduction in any medium, provided that original work is properly cited. Contact author: Nicki A. Dowling E-mail: n.dowling@unimelb.edu.au 
self-esteem, mood, social confidence, social interaction, and quality of life) and subjective ratings of patient satisfaction. The review concluded that most patients were satisfied and had positive psychological outcomes, but that outcomes were most consistently positive for breast reduction and breast augmentation compared to other procedures such as rhinoplasty and facial procedures.

Using more stringent inclusion criteria, Cook et al. (2006) concluded that there was insufficient evidence for enduring improvement in outcomes after any cosmetic surgery procedure. This review identified 22 prospective cohort studies published from 1992 to 2004 that used standardised measures across several domains of outcome, including body image (six studies), self-esteem (seven studies), and mental health (14 studies). Most studies evaluated the outcomes of breast reduction surgery. The findings revealed that pre-operative body image, but not selfesteem and mental health, for breast reduction patients is generally lower than population norms. Although psychological functioning generally improved after breast reduction surgery, evidence of improvement across each domain was weakened by methodological considerations. A small number of rhinoplasty studies also reported mixed post-operative improvement on measures of mental health. No other surgery type received adequate research attention to draw conclusions about their effect across any domain of outcome.

Despite their different approaches, both systematic reviews reveal that studies of psychological outcomes of surgery generally provide low standards of evidence due to significant methodological limitations rather than the existence of negative evidence. These include the absence of exact definitions of the sample, ascertainment bias (e.g., samples from specialist centres), small sample sizes, and lack of valid measures. They also include an absence of comparison or control groups, high rates of refusal to participate, short follow-up periods, and high loss to followup. Moreover, few studies have explored the outcomes of surgery types other than breast reduction or have compared postoperative outcomes for different surgery types.

A UK study conducted by Klassen et al. (1996) evaluated outcomes for 198 patients undergoing several different cosmetic surgery procedures (breast reduction, breast augmentation, other breast surgery, rhinoplasty, abdominoplasty, pinnaplasty) six months after surgery. Outcomes were measured across three domains, including healthrelated quality of life, self-esteem, and mental health. The findings revealed that all patient groups reported statistically significant improvements in quality of life and self-esteem, however only breast reduction patients reported significant improvements in mental health.

A more recent study conducted by von Soest et al. (2009) in Norway investigated the effect of different cosmetic surgical procedures (breast reduction, liposuction/abdominoplasty, and breast augmentation) on body image, self-esteem, and mental health outcomes in a sample of 155 patients. The cosmetic surgery sample displayed lower scores on pre-operative measures of body image and self-esteem, but similar scores on pre-operative measures of mental health, than a representative comparison sample of 838 Norwegian women with no cosmetic surgery experience. There was a significant improvement in body image and selfesteem, but not mental health, after surgery and post-operative measures across all domains did not differ from the comparison sample. Although there were no significant differences between the surgery types in terms of changes in body image, self-esteem, mental health, or postoperative satisfaction, there were trends for greater improvement in appearance evaluation in the breast augmentation group and lower satisfaction in the liposuction/abdominoplasty group. The authors concluded that further methodologically sound research is required to explore changes in psychological dysfunction after undergoing different types of cosmetic surgery. 


\section{Research Aims}

The present study formed part of a larger study that was designed to empirically derive a pre-surgery screening instrument (Dowling, Honigman, \& Jackson, 2010; Honigman et al., 2011; Jackson, Dowling, Honigman, \& Kalus, in press). The first aim of the present study was to explore the psychological outcomes (body image, selfesteem, and mental health) and postoperative satisfaction of cosmetic surgery. It was hypothesized that the cosmetic surgery sample will display (a) higher levels of psychological dysfunction at the pre-operative evaluation than normative samples; (b) lower levels of psychological dysfunction at the post-operative evaluation than the pre-operative evaluation; and (c) comparable levels of psychological dysfunction at the postoperative evaluation than normative samples. The second aim was to compare the outcomes and satisfaction of participants undergoing different surgical procedures. It was hypothesized that breast reduction and breast augmentation patients would display better psychological outcomes and higher levels of postoperative satisfaction than patients undergoing other procedures such as rhinoplasty and facial procedures.

\section{Method}

\section{Participants}

The sample comprised 142 patients (131 females, 11 males) undergoing elective cosmetic surgery in Australia. Most participants underwent rhinoplasty only $(28.2 \%)$ or breast augmentation only $(23.9 \%)$, with smaller proportions undergoing multiple procedures (16.2\%), facial procedures (facelift, blepharoplasty, or otoplasty) only (12.7\%), breast reduction only $(10.6 \%)$, and body procedures (liposuction or abdominoplasty) only (8.5\%). The sample was aged between 19 and 71 years $(M=$ $39.8, S D=10.9$, median $=39.0$ years $)$. Most were born in Australia (73.9\%) and most were employed on a full-time basis $(40.1 \%)$ or part-time basis $(39.3 \%)$. Most participants reported that they were married/cohabiting (50.7\%) or in a noncohabiting relationship (20.4\%).

\section{Measures}

Patients were administered a survey prior to surgery and six months following surgery. The surveys included several wellvalidated standardised measures of body image (appearance evaluation, appearance orientation, and body areas satisfaction), self-esteem, and mental health (psychiatric disturbance, anxiety, depression, and dysmorphic concerns). These measures have been extensively used in research and clinical practice and have been employed in a number of studies evaluating psychological factors in cosmetic surgery (e.g., Castle, Molton, Hoffman, Preston, \& Phillips, 2004; Cunningham \& Feinmann, 1998; Figueroa-Haas, 2007; Kisely, Morkell, Allbrok, Briggs, \& Jovnovic, 2002; Klassen et al., 1996; von Soest et al., 2006).

The Multidimensional Body-Self Relations Questionnaire (MBSRQ: Cash, 2000) is a well-validated self-report inventory for the assessment of body image. Subscales used in the present study were: Appearance Evaluation (7 items: feelings of physical attractiveness and satisfaction with one's looks), Appearance Orientation (12 items: extent of investment in one's appearance), and Body Areas Satisfaction (9 items: satisfaction with discrete aspects of one's appearance) (37). The MBSRQ employs a 5point Likert type response format and displays good internal reliabilities $(\alpha=.73$ to .88) (Cash, 2000). The MBSRQ has been used in a number of cosmetic and correctional surgery studies (e.g., FigueroaHaas, 2007; von Soest et al., 2006). The internal consistencies of each subscale were also good in the pre-operative survey $(\alpha=.79$ to .85$)$ and the post-operative survey ( $\alpha=.79$ to .86 ) of the current study.

The Rosenberg Self-Esteem Scale (RSE: Rosenberg, 1989) is a widely used selfreport questionnaire that is comprised of 10 items that are used to generate a global self-esteem score. The RSE employs a 4point Likert type response format and scores can range from 0 to 30 . The RSE displays high internal consistency $(\alpha=.82)$ 
and item-total correlations in the upper range $(r>.50)$ (Roth, Decker, Herzberg, \& Brähler, 2008). The RSE has been used in a number of cosmetic surgery studies (e.g., Figueroa-Haas, 2007; von Soest et al., 2006). The internal consistency of the RSE was high in both the pre-operative $(\alpha=$ $.86)$ and post-operative survey $(\alpha=.85)$ of the current study.

The General Health Questionnaire (GHQ-30: Goldberg \& Williams, 1988) is a 30 -item measure of state psychiatric disturbance. It employs a 4-point Likert type response format and scores can range from 0 to 90 . The GHQ, in its various forms, has been used in a number of cosmetic surgery studies (Kisely et al., 2002; Klassen et al., 1996). The psychometric properties of the instrument are sound with high measures of reliability (Cronbach's $\alpha=.94$ ) and validity (Lee et al., 1997). The internal consistency of the GHQ-30 was high in both the pre-operative $(\alpha=.90)$ and postoperative survey $(\alpha=.94)$ of the current study.

The Hospital Anxiety and Depression Scale (HADS: Zigmond \& Snaith, 1983) was employed to measure past week generalized anxiety (7 items) and depression (7 items), the latter largely measuring the state of anhedonia. The HADS employs a 4-point Likert type response format and scores for each subscale can range from 0 to 21 . The HADS has been used in a study relating to body dyspmorphic disorder in patients requesting orthognathic surgery (Cunningham \& Feinmann, 1998). The HADS has shown consistently good psychometric properties, with adequate to high internal consistency reliabilities $(\alpha=$ .77 to .82) (Crawford, Henry, Crombie, \& Taylor, 2001). The internal consistency of both subscales was good in the preoperative survey ( $\alpha=.75$ to .84$)$ and postoperative survey ( $\alpha=.79$ to .83 ).

The Dysmorphic Concerns Questionnaire (DCQ: Jorgensen, Castle, Roberts, \& GrothMarnat, 2001) is a 7-item questionnaire measuring the extent of concern with physical appearance. Respondents are asked to respond using a 4-point Likert scale from (0) not at all to (3) much more than most people. The DCQ has been used in a number of cosmetic surgery studies (e.g., Castle et al., 2004; Kisely et al., 2002). The DCQ has demonstrated good psychometric properties, with a Cronbach's alpha of .80 (Jorgensen et al., 2001). Most of the variance $(39 \%)$ is explained by a single factor and it shows strong correlations with measures of depression and social anxiety (Jorgensen et al., 2001). The internal consistency of the DCQ was high in both the pre-operative and post-operative survey of the current study $(\alpha=.85)$.

The post-operative survey also included four items evaluating post-operative satisfaction. These were (1) On a scale of 1 to 10 , how likely do you now think it is, that you have achieved, or will achieve what you were looking for, in having this procedure?; (2) Compared to your expectations, what was the overall result of your surgery? (Better than or as expected and Worse than expected); (3) Overall, would you say that you were satisfied with the outcome of this surgery? (Satisfied and Dissatisfied), and; (4) Compared to your expectations, what was your experience of surgery and recovery? (Better than or as expected and Worse than expected).

\section{Procedure and Data Analyses}

The study was approved by the University of Melbourne Health Sciences Human Ethics Sub-Committee (050782.1). Preoperative surveys were distributed to prospective patients aged 18 years and over of cosmetic surgeons in Melbourne and Sydney (Australia). Pre-operative data was collected from 443 patients. Participants were provided with plain language statements and return of the survey was taken to imply consent. Overall, 142 participants (32.1\%) completed the post-operative survey six months after surgery. Participants completing the postoperative survey were significantly older, $z$ $=-3.73, p<.001, d=0.36$, less likely to be employed full-time, $\chi^{2}(1)=6.88, p=.009$, and reported lower levels of HADS Anxiety, $z=-3.16, p=.003, d=0.35$. 
Missing data, which represented less than five percent and appeared to be at random, was managed using a combination of casewise exclusion for scales with more than $30 \%$ missing data and person mean substitution for scales with less than $30 \%$ missing data. Adjustments were employed to control for multiple comparisons ( $p \leq$ $.01)$, and trends $(p<.05)$ and effect sizes (Cohen's $d$ ) were reported. Differences between those who did and did not complete the post-operative survey were analysed using chi-square analyses, independent samples $t$-tests (for normally distributed data), and Mann Whitney tests (for non-normally distributed data). Onesample $t$-tests were used to compare the pre-operative and post-operative scores of the cosmetic surgery sample with the population norms for each scale. Differences in pre- and post-operative scores were analysed using repeated measures $t$-tests (for normally distributed data) and Wilcoxin signed-ranks tests (for non-normally distributed data). Finally, analyses of covariance were conducted to examine differences in the effectiveness of different surgery types. Excluding participants undergoing multiple operations, only breast augmentation and rhinoplasty had adequate samples for comparison.

\section{Results}

\section{Psychosocial Outcomes of Surgery}

Comparison of pre-operative means to population norms. The cosmetic surgery sample reported significantly lower preoperative MBSRQ Appearance Evaluation, $t$ (124) $=-3.39, p=.001, d=0.32$, and higher DCQ Dysmorphic Concerns, $t(130)=8.47$, $p<.001, d=1.05$, than population norms. Unexpectedly, the cosmetic surgery sample also reported significantly lower MBSRQ Appearance Orientation, $t(124)=-4.28, p<$ $.001, d=0.50$, and HADS Depression, $t$ $(126)=-5.00, p<.001, d=0.65$, than population norms.

Psychological outcomes. The pre- and postoperative survey scores on the standardized measures of psychological functioning are displayed in Table 1. An examination of Table 1 reveals that there was significant improvement on MBSRQ Appearance Evaluation, MBSRQ Body Areas Satisfaction, HADS Anxiety, HADS Depression, and DCQ Dysmorphic Concerns following surgery.

Table 1: Pre- and Post-Operative Survey Scores on Psychological Dysfunction Measures

\begin{tabular}{|c|c|c|c|c|c|c|c|c|}
\hline \multirow[t]{2}{*}{ Measure } & \multirow[t]{2}{*}{$n$} & \multicolumn{2}{|c|}{$\begin{array}{c}\text { Pre- } \\
\text { operative } \\
\text { survey }\end{array}$} & \multicolumn{2}{|c|}{$\begin{array}{c}\text { Post- } \\
\text { operative } \\
\text { survey }\end{array}$} & \multicolumn{3}{|c|}{ t-test/Mann Whitney statistics } \\
\hline & & $M$ & $S D$ & $M$ & $S D$ & Statistic & $p$ & $\begin{array}{c}\text { Effect } \\
\text { size }(d)\end{array}$ \\
\hline MBSRQ Appearance Evaluation & 126 & 3.2 & 0.7 & 3.4 & 0.7 & $t=-4.32$ & $<.001^{* *}$ & 0.29 \\
\hline MBSRQ Appearance Orientation & 125 & 3.7 & 0.6 & 3.7 & 0.6 & $t=1.35$ & .18 & 0.01 \\
\hline MBSRQ Body Areas Satisfaction & 128 & 3.3 & 0.6 & 3.4 & 0.6 & $t=-2.84$ & $.005^{* *}$ & 0.17 \\
\hline Rosenberg Self-esteem & 126 & 22.1 & 4.7 & 22.4 & 4.5 & $t=-0.74$ & .46 & 0.07 \\
\hline GHQ-30 Psychiatric Disturbance & 128 & 22.6 & 8.5 & 21.5 & 9.4 & $Z=-1.11$ & .27 & 0.12 \\
\hline HADS Anxiety & 129 & 5.9 & 3.7 & 5.0 & 3.5 & $Z=-3.12$ & $.002^{* *}$ & 0.25 \\
\hline HADS Depression & 127 & 2.4 & 2.8 & 1.8 & 2.4 & $Z=-2.58$ & $.008^{* *}$ & 0.23 \\
\hline DCQ Dysmorphic Concern & 131 & 7.8 & 4.5 & 6.8 & 4.2 & $t=3.04$ & $.003^{* *}$ & 0.23 \\
\hline
\end{tabular}

${ }^{* *} p \leq .01, * p \leq .05$ 
Comparison of post-operative means to population norms. The cosmetic surgery sample reported significantly lower postoperative MBSRQ Appearance Orientation, $t(124)=-4.28, p<.001, d=0.50$, higher MBSRQ Body Areas Satisfaction, $t$ (127) = 3.56, $p=.001, d=0.40$, lower HADS Anxiety, $t(128)=-3.66, p<.001, d=0.46$, and lower HADS Depression, $t(126)=-$ 8.83, $p<.001, d=1.11$, than population norms. Post-operative DCQ Dysmorphic Concerns scores, however, remained significantly higher, $t(130)=6.38, p<.001$, $d=0.79$, than population norms.

\section{Post-Operative Satisfaction}

Ratings of how likely participants thought it was that they had achieved, or would achieve, what they were looking for in having this procedure ranged from 1 to 10 , with a mean of $7.6(S D=2.4$, median $=8.0)$. Most reported that the overall result of their surgery was better than or as expected $(80 \%)$, overall satisfaction with the outcome of the surgery (83\%), and their experience of surgery and recovery was better than or as expected (85\%).

\section{Differences in Psychological Outcomes and Post-Operative Satisfaction between Surgery Types}

Excluding participants undergoing multiple operations, only breast augmentation and rhinoplasty had adequate samples for comparison. The pre- and post-operative scores on the measures of psychological dysfunction for the breast augmentation and rhinoplasty patients are displayed in Table 2. An examination of Table 2 reveals that breast augmentation patients reported significantly higher improvement on MBSRQ Appearance Evaluation than rhinoplasty patients.

\section{Table 2: Pre- and Post-Operative Psychological Dysfunction for Breast Augmentation and Rhinoplasty Groups}

\begin{tabular}{|c|c|c|c|c|c|c|c|c|}
\hline Measure & \multirow[t]{2}{*}{$n$} & \multicolumn{2}{|c|}{ Pre-operative } & \multicolumn{2}{|c|}{ Post-operative } & \multicolumn{3}{|c|}{ ANCOVA statistics } \\
\hline & & $M$ & $S D$ & $M$ & $S D$ & $\begin{array}{c}\text { Effect } \\
\text { size }(d)\end{array}$ & $F$ & $p$ \\
\hline MBSRQ Appearance Evaluation & & & & & & & 6.34 & $.01^{* *}$ \\
\hline Breast augmentation & 29 & 3.1 & 0.7 & 3.6 & 0.7 & 0.71 & & \\
\hline Rhinoplasty & 37 & 3.3 & 0.7 & 3.4 & 0.6 & 0.15 & & \\
\hline MBSRQ Appearance Orientation & & & & & & & 0.11 & .74 \\
\hline Breast augmentation & 29 & 3.8 & 0.6 & 3.8 & 0.6 & 0.00 & & \\
\hline Rhinoplasty & 37 & 3.7 & 0.6 & 3.7 & 0.6 & 0.00 & & \\
\hline MBSRQ Body Areas Satisfaction & & & & & & & 1.91 & .17 \\
\hline Breast augmentation & 29 & 3.3 & 0.5 & 3.4 & 0.6 & 0.18 & & \\
\hline Rhinoplasty & 37 & 3.3 & 0.7 & 3.4 & 0.6 & 0.15 & & \\
\hline Rosenberg Self-esteem & & & & & & & 1.91 & .17 \\
\hline \begin{tabular}{l|l} 
& Breast augmentation \\
\end{tabular} & 28 & 21.9 & 5.1 & 23.2 & 4.6 & 0.27 & & \\
\hline Rhinoplasty & 37 & 21.7 & 5.1 & 21.6 & 5.0 & 0.02 & & \\
\hline GHQ-30 Psychiatric disturbance & & & & & & & 0.03 & .87 \\
\hline Breast augmentation & 29 & 21.7 & 7.9 & 21.3 & 9.1 & 0.05 & & \\
\hline Rhinoplasty & 37 & 23.6 & 11.1 & 22.6 & 11.7 & 0.09 & & \\
\hline HADS Anxiety & & & & & & & 0.13 & .72 \\
\hline Breast augmentation & 29 & 5.4 & 2.9 & 4.9 & 2.9 & 0.17 & & \\
\hline Rhinoplasty & 38 & 5.8 & 3.9 & 5.4 & 3.9 & 0.10 & & \\
\hline HADS Depression & & & & & & & 2.29 & .14 \\
\hline Breast augmentation & 29 & 2.6 & 3.1 & 1.8 & 2.2 & 0.30 & & \\
\hline Rhinoplasty & 37 & 2.6 & 2.5 & 2.6 & 2.8 & 0.00 & & \\
\hline DCQ Dysmorphic concern & & & & & & & 0.03 & .86 \\
\hline \begin{tabular}{l|l} 
& Breast augmentation \\
\end{tabular} & 29 & 10.7 & 4.5 & 8.9 & 3.9 & 0.43 & & \\
\hline Rhinoplasty & 38 & 8.0 & 3.9 & 7.7 & 3.6 & 0.08 & & \\
\hline
\end{tabular}


Table 3 reveals the post-operative satisfaction for breast augmentation and rhinoplasty patients. An examination of Table 3 reveals that breast augmentation patients reported significantly higher ratings of how likely they thought it was that they had, or would, achieve what they were looking for in having the procedure than rhinoplasty patients. There was also a non-significant trend for a higher proportion of breast augmentation patients to report that the overall result of their surgery was better than or as expected than rhinoplasty patients.

Table 3: Post-Operative Satisfaction for Breast Augmentation and Rhinoplasty Groups

\begin{tabular}{|c|c|c|c|c|c|}
\hline & $n$ & & \multicolumn{3}{|c|}{$t$-test $/ \chi^{2}$ statistics } \\
\hline & & & Statistic & $p$ & Effect size $(d)$ \\
\hline Achievement rating & & & $Z=-2.36$ & $.02 *$ & .11 \\
\hline Breast augmentation & 33 & $8.6(1.3)$ & & & \\
\hline Rhinoplasty & 39 & $7.1(2.7)$ & & & \\
\hline Overall result better than or as expected & & & $\chi^{2}=6.18$ & $.01^{* *}$ & \\
\hline Breast augmentation & 34 & $94.1 \%$ & & & \\
\hline Rhinoplasty & 39 & $71.8 \%$ & & & \\
\hline Satisfied with outcome of surgery & & & $\chi^{2}=3.29$ & .07 & \\
\hline Breast augmentation & 34 & $94.1 \%$ & & & \\
\hline Rhinoplasty & 39 & $79.5 \%$ & & & \\
\hline Experience better than or as expected & & & $\chi^{2}=0.00$ & .99 & \\
\hline Breast augmentation & 34 & $79.4 \%$ & & & \\
\hline Rhinoplasty & 39 & $79.5 \%$ & & & \\
\hline
\end{tabular}

${ }^{* *} p \leq .01,{ }^{*} p \leq .05$

\section{Discussion}

This study, which forms part of a larger study designed to empirically derive a presurgery screening instrument, firstly aimed to explore the psychological outcomes (body image, self-esteem, and mental health) and post-operative satisfaction of cosmetic surgery. The sample was generally a psychologically healthy group on presentation, although they did display low appearance evaluation and high dysmorphic concerns. Six months following surgery, there were high rates of postoperative satisfaction of 80 to $85 \%$ and significant improvements in several dimensions of body image (appearance evaluation and body areas satisfaction) and mental health (anxiety, depression, and dysmorphic concerns), but not self-esteem. Post-operatively, the sample displayed similar or superior psychological functioning than population means, with the exception of dysmorphic concerns. Taken together, these findings are generally consistent with previous findings of improvements in body image (von Soest et al., 2009), but contrast with those that reveal significant improvements in self- esteem across different surgical groups (Klassen et al., 2004; von Soest et al., 2009). They are also not consistent with findings that indices of mental health display the least consistent improvements across different surgical groups (Klassen et al., 2004; von Soest et al., 2009). Although the findings of the current study suggest that cosmetic surgery is generally associated with high rates of patient satisfaction and positive psychological outcomes, it is evident that further research is required to clarify the improvement the degree to which self-esteem and indices of mental health improve after cosmetic surgery.

The second aim of this study was to compare the outcomes and satisfaction of participants undergoing different cosmetic procedures. Breast augmentation patients reported significantly higher improvement on appearance evaluation, significantly higher ratings of how likely they thought it was that they had, or would, achieve what they were looking for, and a trend to report that the overall result was better than or as expected than rhinoplasty patients. These findings are consistent with the conclusion of the review conducted by Honigman et al. 
(2004) that breast augmentation, which is associated with improvements in attractiveness and body image satisfaction, displays superior patient outcomes and satisfaction than rhinoplasty procedures. They are also consistent with the findings of von Soest et al., (2009) who reported trends for greater improvement in appearance evaluation and higher postoperative satisfaction for patients undergoing breast augmentation than body procedures.

Pre-operative screening is recommended to identify the minority of patients, including those undergoing rhinoplasty, who will report poorer outcomes and postoperative dissatisfaction. We have developed the PREFACE ${ }^{\odot}$, a brief and objective screening protocol to assist practitioners in identifying elective facial cosmetic surgery and dentistry patients who may benefit from pre-operative counseling (Honigman et al., 2011). This screening tool can be readily adapted for other cosmetic surgery populations.

Despite the generally positive outcomes of patients undergoing cosmetic surgery in this study, the findings should be interpreted with caution due to several methodological considerations. Firstly, the sample comprised a relatively small sample that was compared with population norms. Comparison with population norms fails to control for local variations in populations or involvement in healthcare (Cook et al., 2006) and the small sample precluded comparison of all different types of surgery. Secondly, the six-month follow-up period may under-estimate benefits by reflecting cognitive dissonance, whereby patients report positive outcomes to fit the decision to undergo cosmetic surgery, or over-estimate benefits by allowing insufficient time for the interaction of changes that would lead to psychological improvement (Cook et al., 2006). Finally, similar to most studies in cosmetic surgery outcomes, there was a high loss to followup in this study, which may result in a sample bias of patients with superior outcomes agreeing to participate (Honigman et al., 2004). It is therefore recommended that future research replicates this study using larger samples, use of control groups with physical indications for surgery, longer follow-up periods, and high follow-up rates in order to fully establish the impact of cosmetic surgery on body image, self-esteem, and mental health outcomes.

\section{References}

Cash, T. F. (2000). 'The Multidimensional Body-Self Relations Questionnaire Users' Manual, 2000,' Available at: http://www.body-images.com.

Castle, D. J., Molton, M., Hoffman, K., Preston, N. J. \& Phillips, K. A. (2004). "Correlates of Dysmorphic concern in People Seeking Cosmetic Enhancement," Australian and New Zealand Journal of Psychiatry, 38(6), 439-444.

Cook, S. A., Rosser, R. \& Salmon, P. (2006). "Is Cosmetic Surgery an Effective Psychotherapeutic Intervention?: A Systematic Review of the Evidence," Journal of Plastic, Reconstructive and Aesthetic Surgery, 59 (11) 1133-1151.

Crawford, J. R., Henry, J. D., Crombie, C. \& Taylor, E. P. (2001). "Normative Data for the HADS from a Large Non-Clinical Sample," British Journal of Clinical Psychology, 40 (4) 429-434.

Cunningham, S. J. \& Feinmann, C. (1998). "Psychological Assessment of Patients Requesting Orthognathic Surgery and the Relevance of Body Dysmorphic Disorder," British Journal of Orthodontics, 25(4), 293298.

Dowling, N. A., Honigman, R. J. \& Jackson, A. C. (2010). "The Male Cosmetic Surgery Patient: A Matched Sample Gender Analysis of Elective Cosmetic Surgery and Cosmetic Dentistry Patients", Annals of Plastic Surgery, 64 (6) 726-731.

Figueroa-Haas, C. L. (2007). "Effect of Breast Augmentation Mammoplasty on Self-Esteem and Sexuality: A Quantitative Analysis," Plastic Surgical Nursing, 27(1), 16-36.Goldberg, D. P., and Williams, P. (1988) A User's Guide to the General 
Health Questionnaire, NFER-Nelson, Windsor.

Honigman, R. J., Jackson, A. C. \& Dowling, N. A. (2011). "The PreFACEC: A PreOperative Psychosocial Screen for Elective Facial Cosmetic Surgery and Cosmetic Dentistry Patients," Annals of Plastic Surgery, 66 (1) 16-23.

Honigman, R. J., Phillips, K. A. \& Castle, D. J. (2004). "A Review Of Psychosocial Outcomes for Patients Seeking Cosmetic Surgery," Plastic and Reconstructive Surgery, 113 (4) 1229-1237.

Jackson, A. C., Dowling, N. A., Honigman, R. \& Kalus, A. (in press). 'The Experience of Teasing in Elective Cosmetic Surgery Patients,' Behavioral Medicine.

Jorgensen, L., Castle, D., Roberts, C. \& Groth-Marnat, G. (2001). "A Clinical Validation of the Dysmorphic Concern Questionnaire," Australian and New Zealand Journal of Psychiatry, 35 (1) 124128.

Kisely, S., Morkell, D., Allbrok, B., Briggs, P. \& Jovnovic, J. (2002). "Factors Associated with Dysmorphic Concern and Psychiatric Morbidity in Plastic Surgery Outpatients," Australian and New Zealand Journal of Psychiatry, 36(1), 121-126.

Klassen, A., Jenkinson, C., Fitzpatrick, R. \& Goodacre, T. (1996). 'Patients' Health Related Quality of Life before and after Aesthetic Surgery," British Journal of Plastic Surgery, 49 (7) 433-438.

Lee, D. T. S., Wong, C. K., Ungvari, G. S., Cheung, L. P., Haines, C. J. \& Chung, T. K. H. (1997). "Screening Psychiatric Morbidity after Miscarriage: Application of the 30Item General Health Questionnaire and the Edinburgh Postnatal Depression Scale," Psychosomatic Medicine, 59 (2) 207-210.

Rosenberg, M. (1989). Society and the Adolescent Self-image, Wesleyan University Press, Middletown, CT, USA.
Roth, M., Decker, O., Herzberg, P. Y. \& Brähler, E. (2008). "Dimensionality and Norms of the Rosenberg Self-Esteem Scale in a German General Population Sample," European Journal of Psychological Assessment, 24 (3) 190-197.

Von Soest, T., Kvalem, I. L., Roald, H. E. \& Skolleborg, K. C. (2009). "The Effects of Cosmetic Surgery on Body Image, SelfEsteem, and Psychological Problems," Journal of Plastic, Reconstructive and Aesthetic Surgery, 62 (10) 1238-1244.

Zigmond, A. S. \& Snaith, R. P. (1983). "The Hospital Anxiety and Depression Scale," Acta Psychiatrica Scandinavica, 67 (6) 361370. 